\title{
Functional Decomposition of Polynomials
}

\author{
Joachim von zur Gathen* \\ Dexter Kozen ${ }^{\dagger}$ \\ Susan Landau ${ }^{\ddagger}$
}

87-851

July 1987

Department of Computer Science

Cornell University

Ithaca, New York 14853-7501

\footnotetext{
* Supported by NSERC grant 3-650-126-40. Part of this work was done during a visit to Universität des Saarlandes, Saarbrücken.

†Supported by NSF grant DCR-8602663.

'Supported by NSF grants DCR-8402175 and DCR-8301766.
} 



\title{
Functional Decomposition of Polynomials
}

\author{
Joachim von zur Gathen* \\ Department of Computer Science \\ University of Toronto \\ Toronto, Ontario M5S 1A4, Canada
}

\author{
Dexter Kozen $^{\dagger}$ \\ Department of Computer Science \\ Cornell University \\ Ithaca, New York 14853
}

\author{
Susan Landau ${ }^{\ddagger}$ \\ Department of Mathematics \\ Wesleyan University \\ Middletown, Connecticut 06457
}

\section{Introduction}

If $F$ is a commutative ring and $g, h \in F[x]$, then $f=g \circ h \in F[x]$ is their (functional) composition, and $(g, h)$ is a (functional) decomposition of $f$. Given $f \in F[x]$, there exists an essentially unique complete decomposition $f=f_{1} \circ f_{2} \circ \cdots \circ f_{k}$, where $f_{1}, \ldots, f_{k} \in F[x]$ are indecomposable. This result is valid if $F$ is a field whose characteristic does not divide the degree of $f$.

This paper deals with the following decomposition problem: given $f \in F[x]$ of degree $n$, and $r, s \in \mathbf{N}$ with $n=r s$ and $r, s>1$, decide whether there exist $g, h \in F[x]$ of degree $r, s$ respectively, such that $f=g \circ h$. If so, determine the coefficients of $g$ and $h$.

For some time, this problem was considered to be computationally hard: a cryptographic protocol was based on its supposed intractability [5]. Barton and Zippel [3] and Alagar and Thanh [1] gave exponential-time algorithms for it (in characteristic zero).

In $\S 2$, we present fast sequential and parallel algorithms for this problem. In the "tame" case (when the characteristic $p$ of $F$ does not divide $r$ ), we present a sequential algorithm requiring time $O\left(n \log ^{2} n \log \log n\right)$, and $O\left(n \log ^{2} n\right)$ if $F$ supports a Fast Fourier Transform. We show that the problem is in $N C$, and give a depth-optimal $O(\log n)$-depth circuit for it. In addition, we show that the complete decomposition of $f$ can be computed in sequential $O\left(n^{1+\epsilon}\right)$ or parallel $O(\log n)$ time.

\footnotetext{
*Supported by NSERC grant 3-650-126-40. Part of this work was done during a visit to Universität des Saarlandes, Saarbrücken.

†Supported by NSF grant DCR-8602663.

${ }^{\ddagger}$ Supported by NSF grants DCR-8402175 and DCR-8301766.
} 
In $\S 3$, we consider the "wild" case (no restrictions on the characteristic of $F$ ). We give a new structure theorem which gives necessary and sufficient conditions for testing decomposability over $F$. The decomposition problem is shown to be reducible to the problem of factoring univariate polynomials over $F$. We obtain a range of results, from undecidability over sufficiently general fields to fast sequential and parallel algorithms over finite fields.

A version of the algorithm of Theorem 1 below has been implemented $[2,6]$ and compares favorably with [3]. Dickerson [9] has extended some of these results to multivariate polynomials.

We should give a brief history of the research behind this joint paper. Kozen and Landau [18] gave the first polynomial-time sequential and $N C$ algorithms for this problem in the tame case. The time bounds were $O\left(n^{3}\right)$ sequential, $O\left(n^{2}\right)$ if $F$ supports an FFT, and $O\left(\log ^{2} n\right)$ parallel. They also presented the structure theorem (Theorem 9 ), reducing the problem in the wild case to factorization, and gave an $O\left(n^{\log n}\right)$ algorithm for the decomposition of irreducible polynomials over general fields admitting a polynomial-time factorization algorithm, and an $N C$ algorithm for irreducible polynomials over finite fields.

Based on the algorithm of $[18 \mid$, von zur Gathen [17] improved the bounds in the tame case to those stated above. These results are presented in $\S 2$. He also gave an improved algorithm for the wild case, yielding a polynomial-time reduction to factorization of polynomials, and observed undecidability over sufficiently general fields. These results are presented in $§ 3$.

\section{Fast Decomposition in the tame case}

We consider the following decomposition problem $\operatorname{DEC}_{n, r}^{F}$. We have a field $F$, integers $n, r \in \mathbf{N}$ with $r$ dividing $n$, and $f \in F[x]$ of degree $n$. Let $s=n / r$. The problem is to decide whether there exist $g, h \in F[x]$ of degrees $r, s>1$, respectively, such that $f=g \circ h$, and, in the affirmative case, to determine the coefficients of $g$ and $h$. We say $f$ is indecomposable if no such $g$ and $h$ exist for any $r, s$. The "tame" case is when the characteristic $p$ of $F$ does not divide $r$. This section deals only with the tame case.

For the question of uniqueness, we note the following three types of ambiguous decompositions. For any $u \in F[x], \alpha \in F$, and $r, m \geq 2$ we have

$$
\begin{gathered}
u \circ(x-\alpha) \circ(x+\alpha)=u \\
x^{r} \circ\left(x^{m} \cdot u\left(x^{r}\right)\right)=\left(x^{m} \cdot u^{r}\right) \circ x^{r} \\
T_{r} \circ T_{m}=T_{m} \circ T_{r}
\end{gathered}
$$

where $T_{r}, T_{m}$ are Chebyshev polynomials. Ritt's first theorem states that a decomposition $f=f_{1} \circ \cdots \circ f_{k}$ with $f_{1}, \ldots, f_{k}$ indecomposable is unique up to these ambiguities, i.e., that any two complete decompositions can be obtained from each other using these equalities ([20] for $F=\mathbf{C},[11]$ for $p=0,[12]$ for $p=0$ or $p>n$ ).

Decompositions are intimately related to the intermediate fields between $F(f)$ and $F(x)$ $[10,20]$ and between $F$ and a splitting field of $f$ (see Theorem 9 ). 
If $f=g \circ h$ and $a_{n}, c_{s}$ are the leading coefficients of $f, h$, respectively, then

$$
\frac{f}{a_{n}}=\left(\frac{1}{a_{n}} g\left(c_{s} x\right)\right) \circ \frac{h}{c_{s}}
$$

is a decomposition of a monic polynomial into monic polynomials, and so we can assume that $f, g$, and $h$ are monic, and furthermore that $h(0)=0$. Denoting by $P \subseteq F[x]$ the set of monic polynomials, we consider the relation

$$
\operatorname{DEC}_{n, r}^{F}=\left\{(f,(g, h)) \in P \times P^{2} \mid f=g \circ h, \operatorname{deg} f=n, \operatorname{deg} g=r, \text { and } h(0)=0\right\} .
$$

In the tame case, for every $f$ there exists at most one such $(g, h)$, so that we can view $\mathrm{DEC}_{n, r}^{F}$ as a partial function $P \rightarrow P^{2}$. Furthermore, the problem is rational, i.e., if there exists a field extension $K \supseteq F$ and $(f,(g, h)) \in \operatorname{DEC}_{n, r}^{K}$, then in fact $g, h \in F[x][12,19]$. Both facts may fail in the "wild" case; see Example 7.

Let $M=M_{F}: \mathbf{N} \rightarrow \mathbf{R}$ be such that the product of two polynomials in $F[x]$ of degree at most $n$ can be computed with $O(M(n))$ arithmetic operations. We can choose $M(n)=$ $n \log n \log \log n[21]$, and $M(n)=n \log n$ if $F$ supports a Fast Fourier Transform.

Theorem 1 Over any field $F$, the decomposition problem $\operatorname{DEC}_{n, r}^{F}$, with char $(F)$ not dividing $r$, can be computed with $O(M(n) \log n)$ field operations.

Proof. Let $f \in F[x]$ be monic of degree $n=r s$. We look for a decomposition $f=g \circ h$, with $g, h$ monic. We first compute the unique candidate $h$, using that $f$ and $h^{r}$ agree on the highest $s$ terms, i.e. $\operatorname{deg}\left(f-h^{r}\right) \leq n-s$. Writing $f=x^{n}+a_{n-1} x^{n-1}+\cdots+a_{0}$, we let $\tilde{f}=a_{0} x^{n}+\cdots+a_{n-1} x+1=x^{n} \cdot f\left(\frac{1}{x}\right)$ be the reversal of $f$, and similarly $\tilde{h}=x^{s} \cdot h\left(\frac{1}{x}\right)$. Then

$$
\begin{aligned}
& x^{n} h\left(\frac{1}{x}\right)^{r}=\left(x^{s} h\left(\frac{1}{x}\right)\right)^{r}=\tilde{h}^{r} \\
& \operatorname{deg}\left(f-h^{r}\right) \leq n-s \leftrightarrow x^{n} \cdot\left(\left(f-h^{r}\right)\left(\frac{1}{x}\right)\right) \equiv 0 \bmod x^{s} \\
& \leftrightarrow f-\tilde{h}^{r} \equiv 0 \bmod x^{s} .
\end{aligned}
$$

By [4], $\tilde{h} \bmod x^{s}$ can be computed with $O(M(n))$ operations. We obtain $h$ from $\tilde{h}$ by reversing the coefficient sequence and setting the constant coefficient to zero.

Now we compute the coefficients $b_{i}$ of $g$ as a Taylor expansion of $f$ in $h: f=\sum_{0 \leq i \leq r} b_{i} h^{i}$ with $b_{i} \in F[x]$ of degree less than $s$, and return $g=\sum_{0 \leq i \leq r} b_{i} x^{i} \in F[x]$ if each $b_{i} \in F$, and otherwise conclude that no decomposition exists.

Corollary 2 Let $\epsilon>0$. If $\operatorname{char}(F)$ does not divide the degree $n$ of $f$, a complete decomposition of $f$ into indecomposable polynomials can be computed with $O\left(n^{1+\epsilon}\right)$ operations.

Open Question 3 Is it possible to improve the running time further, say to $O(M(n))$ ? 
Remark 4 We have stated Theorem 1 only for the case of a field $F$. It actually works for an arbitrary commutative ring $F$, provided that $r$ is a unit in $F$.

Kozen and Landau observed that the general parallelization technique of $[22]$ applies to their construction, and obtained an arithmetic algorithm of depth $O\left(\log ^{2} n\right)$ in the tame case. Using fast parallel arithmetic for polynomials (see [15]), one finds the following results of order-optimal depth.

Theorem 5 Over any field $F$, the decomposition problem $\operatorname{DEC}_{n, r}^{F}$, with char $(F)$ not dividing $r$, can be computed on an arithmetic network over $F$ of depth $O(\log n)$.

Corollary 6 If char $(F)$ does not divide the degree $n$ of $f$, a complete decomposition of $f$ into indecomposable polynomials can be computed in depth $O(\log n)$.

\section{Decomposition in the wild case}

The literature contains no algorithm to solve the decomposition problem DEC n,r $^{F}$ in the "wild" case, when the characteristic divides $r$. In this section, we present a reduction of the problem to factoring univariate polynomials. We obtain results at four different levels, from worst (undecidable) to best (polynomial time and poly-logarithmic depth). The first two negative results are meant to explain the restrictions we impose in the positive results.

1. The decomposition problem is undecidable in general.

2. If $F$ is not finitely generated over its prime field, decomposition may require algebraic field extensions of $F$ of exponential degree.

3. If $F$ is finitely generated, we have a polynomial-time algorithm.

4. If $F$ is finite, we have a fast sequential $\left(O\left(n^{3}\right)\right)$ and a fast parallel $\left(O\left(\log ^{2} n\right)\right)$ algorithm.

The results of this section are from [17], except for Definition 1 and Theorem 9, which are from [18].

In view of $\S 2$, we only have to consider a field $F$ of characteristic $p>0$, and $\mathrm{DEC}_{n, r}^{F}$ with $p$ dividing $r$. In the wild case, both the uniqueness and the rationality of decomposition may fail $[10,13]$. Here are some simple examples of this wild behavior, which also illustrate the general algorithm.

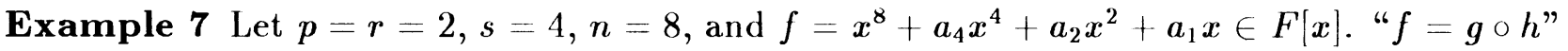
is equivalent to:

$$
a_{4}=c_{2}^{2}+b_{1}, \quad a_{2}=c_{1}^{2}+b_{1} c_{2}, \quad a_{1}=b_{1} c_{1} .
$$


The algorithm takes the first equation in two unknowns and solves for $c_{2}$ in terms of an indeterminate $z$; later we find an equation for $z$ alone and substitute its solutions for $b_{1} . c_{1}$ is similarly determined from the second equation:

$$
c_{2}=\sqrt{a_{4}}+\sqrt{z}, \quad c_{1}=\sqrt{a_{2}}+\sqrt{z}\left(\sqrt[4]{a_{4}}+\sqrt[4]{z}\right) .
$$

The third equation, taken to the fourth power, then yields:

$$
z^{7}+a_{4} z^{6}+a_{2}^{2} z^{4}+a_{1}^{4}=0 .
$$

We take $b_{1}$ to be any of the solutions, and substitute to obtain the corresponding $c_{1}, c_{2}$.

Example 8 Let $F=\mathbf{Z}_{3}, f=x^{6}+x^{4}-x^{3}+x^{2}+x \in F[x], h=x^{2}+c x, g=x^{3}+b_{2} x^{2}+b_{1} x$. Then $v=z^{3}+2 z+1 \in F[z]$ has no linear factors, and hence is irreducible. The high order terms of $g \circ h$ are $x^{6}+b_{2} x^{4}+\left(c^{3}+2 b_{2} c\right) x^{3}$; if $b_{2}$ and $c$ are in $F$, then $f \neq g \circ h$. However, let $\gamma \in G F(27)$ be such that $v(\gamma)=0, c=\gamma, b_{2}=1, b_{1}=-\gamma^{2}+1$. Then $f=g \circ h$. This shows that decompositions may exist in algebraic extensions without existing in the ground field. Also, the three conjugate solutions obtained in this way are not "essentially equivalent"; thus Ritt's first theorem also fails in this case.

Our first result is a structure theorem for decomposability. Let $F$ be a field of arbitrary characteristic. Let $f \in F[x]$ be monic of degree $n=r s$, not necessarily irreducible or separable. Let $\hat{F}$ denote the splitting field of $f$. Let $\mathcal{G}$ denote the Galois group of $\hat{F}$ over $F$. The following definition reduces to the usual notion of block decomposition for $f$ irreducible and separable.

Definition 1 A block decomposition for $f$ is a multiset $\Delta$ of multisets of elements of $\hat{F}$ such that

1. $f(x)=\prod_{A \in \Delta} \prod_{\alpha \in A}(x-\alpha)$

2. if $\alpha \in A \in \Delta, \beta \in B \in \Delta$, and $\sigma \in \mathcal{G}$ such that $\sigma(\alpha)=\beta$, then

$$
B=\{\sigma(\gamma) \mid \gamma \in A\}
$$

A block decomposition $\Delta$ is an $r \times s$ block decomposition if $|\Delta|=r$ and $|A|=s$ for all $A \in \Delta$.

Let $c_{k}^{m}$ denote the $k^{\text {th }}$ elementary symmetric function on $m$-element multisets of elements of $F$ :

$$
c_{k}^{m}(A)=\sum_{B \subseteq A,|B|=k} \prod B
$$

By convention, $c_{0}^{m}=1$.

Theorem 9 Let $f \in F[x]$ be monic of degree $n=r s$. The following two statements are equivalent: 
1. $f=g \circ h$ for some $g, h \in F[x]$ of degree $r$ and $s$, respectively;

2. there exists an $r \times s$ block decomposition $\Delta$ for $f$ such that

$$
c_{k}^{s}(A)=c_{k}^{s}(B) \in F, \text { for all } A, B \in \Delta, 0 \leq k \leq s-1 .
$$

If $f$ is irreducible, then we need only check the condition of Theorem $9(2)$ for one $A \in \Delta$; if it holds for one, then it holds for all, since $\mathcal{G}$ is transitive on $\Delta$. The coefficients of $h$ will be the $c_{k}^{s}(A), 1 \leq k \leq s-1$. The constant coefficient of $h$ is 0 , without loss of generality. The roots of $g$ are $c_{s}^{s}(A), A \in \Delta$. The coefficients of $g$ may be obtained by solving a triangular linear system, or by the method of Theorem 1 .

Theorem 9(2) gives an algebraic condition that can be used to test decomposability of any $f$ over any field $F$, provided one can factor over $F$ and thereby construct the splitting field of $f$. The complexity of the algorithm depends on the complexity of factoring over $F$.

In order to solve $\mathrm{DEC}_{n, r}^{F}$, it would be sufficient to compute the decompositions in $F[x]$. However, a satisfactory solution to the decomposition problem should also return the decompositions over algebraic extensions. We define $\overline{\mathrm{DEC}}_{n, r}^{F}$ to be the computational problem of testing whether a monic input $f \in F[x]$ of degree $n=r s$ has an "absolute" decomposition $f=g \circ h$, where $g, h \in K[x]$ are monic polynomials of degree $r, s$, respectively, over an algebraic closure $K$ of $F$. If such a decomposition exists, we also have to compute some standard representations of all decompositions.

Theorem 10 Let $F$ be any field. The problem $\overline{\mathrm{DEC}}_{n, r}^{F}$ of decomposing polynomials over $F$ is polynomial-time reducible to the problem of factoring univariate polynomials of degree less than $n$ over $F$.

Since polynomial-time factorization algorithms are available over finitely generated fields [7], we have:

Corollary 11 Over a finitely generated field $F, \overline{\mathrm{DEC}}_{n, r}^{F}$ can be computed in polynomial time.

The following undecidability result works over a computable field [14], has inputs encoded over a finite alphabet, and the Turing machine as model of computation. The arithmetic operations and tests are computable.

Theorem 12 There exists a field $F$ such that $\mathrm{DEC}_{n, r}^{F}$ is undecidable.

If $f(0)=0$ and $f=g \circ h$, then $h$ is a nontrivial factor of $f$. However, in the tame case the decomposition problem can be solved without recourse to factoring. In the wild case, our algorithm does use a factoring routine. Is this really necessary?

For an affirmative answer, we fix a prime $p$, and for simplicity, only consider $F=\mathbf{Z}_{p}$. We call a polynomial $w=\sum w_{i} z^{i} \in F[z]$ "special" if it has degree $1+p+\cdots+p^{e}$ for some $e \geq 1$, and

$$
w_{i} \neq 0 \rightarrow \exists j \leq e+1 \quad i=p^{j}+p^{j+1}+\cdots+p^{e}
$$


The polynomial

$$
w=z^{7}+a_{4} z^{6}+a_{2}^{2} z^{4}+a_{1}^{4}
$$

from the Example 7 is special, with $p=e=2$. It is conjectured that factoring special polynomials is essentially as hard as factoring general polynomials, and that this conjecture is hard to prove. In any case, we have:

Theorem 13 The problem of factoring special polynomials is linear-time reducible to the decomposition problem.

We now work out the cost for decomposition over finite fields. We have a field $F$ with $p^{m}$ elements. We then consider algorithms using arithmetic operations over $\mathbf{Z}_{p}$, assume that $F=\mathbf{Z}_{p}[t] /(v)$ is represented by an irreducible polynomial $v \in \mathbf{Z}_{p}[t]$ of degree $m$, and elements of $F$ represented by vectors in $\mathbf{Z}_{p}^{m}$. Let $\tau$ be an exponent for matrix multiplication over $F$, so that the product of two $d \times d$ matrices over $F$ can be computed with $O\left(d^{\tau}\right)$ operations in $F$. [8] shows $\tau<2.38$.

Theorem 13 noted that factorization of special polynomials is reducible to decomposition. All known methods for factoring, based on Berlekamp's (deterministic) algorithm, require linear algebra and thus $\Omega\left((m n)^{\tau}\right)$ operations (for optimal $\tau$ ). It turns out that all complete decompositions can be computed in essentially the same time bound; any improvement would therefore be major progress. The standard algorithms for factoring univariate polynomials (see e.g. $[16]$ ) yield the following bounds.

Theorem 14 Let $F$ be a finite field with $p^{m}$ elements, $p=\operatorname{char}(F), q$ the largest power of $p$ dividing $r$. Then $\overline{\mathrm{DEC}}_{n, r}^{F}$ can be solved with $O\left((m n)^{\tau}\right)$ operations in $\mathbf{Z}_{p}$, and with $O(M(n) \log n M(m))$ operations, if $q$ does not divide $s=n / r$.

Corollary 15 Let $\epsilon>0$. Absolute indecomposability of polynomials of degree at most $n$ over $G F\left(p^{m}\right)$ can be tested with $O\left((m n)^{\tau+\epsilon}\right)$ operations.

Corollary 16 If $F$ is a finite field with $p^{m}$ elements and $p=\operatorname{char}(F)$, then $\overline{\mathrm{DEC}}_{n, r}^{F}$ can be solved on an arithmetic network over $\mathbf{Z}_{p}$ of depth $O\left(\log ^{2}(m n)\right)$ and size $(m n)^{O(1)}$.

Recall that a decomposition $f=f_{1} \circ \cdots \circ f_{k}$ is complete if each $f_{i}$ is indecomposable. Since $f_{k}, f_{k-1} \circ f_{k}, \cdots$ may be over larger and larger fields, it is somewhat surprising that we can calculate all complete absolute decompositions of a polynomial (i.e. those over an algebraic closure) quickly.

Theorem 17 Let $F$ be a finite field with $p^{m}$ elements and characteristic $p, \epsilon>0$, and $f \in F[x]$ of degree $n$. For $p$ and $n$ sufficiently large, all complete decompositions of $f$ over $F$ and all complete absolute decompositions can be computed with $O\left((m n)^{\tau+\epsilon}\right)$ operations in $F$. If $p^{2} V_{n}$, they can be computed with $O\left((m n)^{1+\epsilon}\right)$ operations. 
In the preceding theorem, $p$ may have to be large. For small $p$, we have:

Corollary 18 Let $p$ be a prime, $F$ a finite field with $p^{m}$ elements and characteristic $p$. All complete absolute decompositions of a polynomial in $F[x]$ of degree at most $n$ can be computed with $O\left(m^{3} n^{7}\right)$ operations in $\mathbf{Z}_{p}$.

Further details, proofs, and precise bounds may be found in the journal versions of this paper.

\section{Acknowledgments}

We are grateful to Arash Baratloo of Cornell and Bruce Char of the University of Waterloo for their implementation of the algorithm of Theorem 1.

\section{References}

[1] V.S. Alagar and M. Thanh, "Fast polynomial decomposition algorithms," Proc. EUROCAL85, Lect. Notes in Comput. Sci. 204, Springer-Verlag, Heidelberg, 1985, 150153.

[2] A. Baratloo, private communication, Cornell Univ., December 1986.

[3] D.R. Barton and R.E. Zippel, "Polynomial decomposition algorithms," J. Symb. Comp. 1 (1985), 159-168.

[4] R.P. Brent and H.T.Kung, "Fast algorithms for manipulating formal power series," J. Assoc. Comput. Mach. 25 (1978), 581-595.

[5] J.J. Cade, "A public key cipher which allows signatures," Proc. 2nd SIAM Conf. on Appl. Linear Algebra, Raleigh, 1985.

[6] B. Char, private communication, Univ. of Waterloo, December 1986.

[7] A.L. Chistov and D.Yu. Grigoryev, "Polynomial-time factoring of the multivariable polynomials over a global field," LOMI preprint E-5-82, Leningrad, 1982.

[8] D. Coppersmith and S. Winograd, "Matrix multiplication via arithmetic progressions," Proc. 19th ACM Symp. Theory of Comput., New York, 1987, 1-6.

[9] M. Dickerson, "Polynomial decomposition algorithms for multivariate polynomials," Tech. Rep. TR87-826, Dept. Comput. Sci, Cornell Univ., April 1987.

[10] F. Dorey and G. Whaples, "Prime and composite polynomials," J. Algebra 28 (1974), 88-101. 
[11] H.T. Engstrom, "Polynomial substitutions," Amer. J. Math 63 (1941), 249-255.

[12] M.D. Fried and R.E. MacRae, "On the invariance of chains of fields," Ill. J. Math. 13 (1969), 165-171.

[13] M.D. Fried and R.E. MacRae, "On curves with separated variables," Math. Ann. 180 (1969), 220-226.

[14] A. Fröhlich and J.C. Shepherdson, "Effective procedures in field theory," Phil. Trans. Royal Soc., Ser. A 248 (1955-56), 407-432.

[15] J. von zur Gathen, "Parallel arithmetic computations: a survey," Proc. 12th Int. Symp. Math. Found. Comput. Sci., Bratislava, Lect. Notes in Comput. Sci. 233, Springer, 1986, 93-112.

[16] J. von zur Gathen, "Factoring polynomials and primitive elements for special primes," Theor. Comput. Sci. 51 (1987), to appear.

[17] J. von zur Gathen, "Functional decomposition of polynomials," Tech. Rep., Sonderforschungsbereich 124, Universität Saarbrücken, July 1987.

[18] D. Kozen and S. Landau, "Polynomial decomposition algorithms," Tech. Rep. TR86-773, Dept. Comput. Sci., Cornell Univ., August 1986; J. Symb. Comp., to appear.

[19] H. Levi, "Composite polynomials with coefficients in an arbitrary field of characteristic zero," Amer. J. Math. 64 (1942), 389-400.

[20] J.F. Ritt, "Prime and composite polynomials," Trans. Amer. Math. Soc. 23 (1922), 51-66.

[21] A. Schönhage, "Schnelle Multiplikation von Polynomen über Körpern der Charakteristik 2," Acta Informatica 7 (1977), 395-398.

[22] L.G. Valiant, S. Skyum, S. Berkowitz, and C. Rackoff, "Fast parallel computation of polynomials using few processors," SIAM J. Comput. 12:4 (1983), 641-644. 